\title{
Percepción del proceso de inclusión en educación básica regular en docentes del nivel primario de Lima norte
}

\section{Perception of the process of inclusion in regular basic education among teachers in the primary level of Lima north}

\author{
Gina Estrella-Serón \\ Universidad César Vallejo, Lima, Perú \\ Orcid: https://orcid.org/0000-0002-3859-9755
}

William Mory-Chiparra

Universidad César Vallejo, Lima, Perú

Orcid: https://orcid.org/0000-0003-1404-4605

Noemí Mendoza-Retamozo

Universidad César Vallejo, Lima, Perú

Orcid: https://orcid.org/0000-0003-1865-0338

Isabel Menacho-Vargas

Universidad César Vallejo, Lima, Perú

Orcid: https://orcid.org/0000-0001-6246-4618

Claudia Quispe-Chero

Universidad Privada San Juan Bautista, Lima, Perú

Orcid: https://orcid.org/0000-0002-9957-6530

Received 02-24-20 Revised 05-25-20 Accepted 08-08-20 On line 10-25-20

*Correspondence

Email: gestrellasaanee@gmail.com
Cite as:

Estrella-Serón, G., Mory-Chiparra, W., Mendoza-Retamozo, N., Menacho-Vargas, I., \& Quispe-Chero, C. (2020). Percepción del proceso de inclusión en educación básica regular en docentes del nivel primario de Lima norte. Propósitos y Representaciones, 8(3), e429. doi: http://dx.doi.org/10.20511/pyr2020.v8n3.784 


\section{Resumen}

La posibilidad de que las instituciones educativas de todo nivel se orienten a buscar consolidar mecanismos de inclusión es el ideal de la equidad en educación. Por ello el presente trabajo se optó por el diseño descriptivo no experimental, orientado al análisis comparativo de la percepción del proceso de inclusión en dos instituciones educativas del nivel básico en dos distritos de Lima Norte. La muestra estuvo conformada por 120 docentes del nivel de educación primario, a los cuales se les aplicó el cuestionario adaptado de indicadores de Inclusión - Bristol (2019), conformado por tres dimensiones: Creando culturas inclusivas, con 13 ítems, Realizando políticas inclusivas, con 11 ítems y Desarrollando prácticas inclusivas con 16 ítems; el cual fue validado por juicio de expertos. Los resultados indicaron que existieron diferencias significativas en el nivel de percepción del proceso de inclusión entre los docentes de ambas locaciones, así como variaciones en la percepción de las dimensiones planteadas. Se concluyó que la existencia de diferencias en la percepción de inclusión de los docentes de los dos distritos se ha debido a las posturas de los docentes frente a la política institucional y su percepción del proceso de inclusión.

Palabras clave: inclusión, políticas inclusivas, cultura inclusiva, diversidad, igualdad de oportunidades.

\section{Summary}

The possibility for educational institutions of all levels to seek to consolidate inclusion mechanisms is the ideal of equity in education. For this reason, the present work opted for the non-experimental descriptive design, aimed at comparative analysis of the perception of the inclusion process in two basic level educational institutions in two districts of North Lima. The sample was made up of 120 teachers from the primary education level, to which the adapted questionnaire of indicators of Inclusion - Bristol (2019) was applied, made up of three dimensions: Creating inclusive cultures, with 13 items, Carrying out inclusive policies, with 11 items and Developing inclusive practices with 16 items; which was validated by expert judgment. The results indicated that there were significant differences in the level of perception of the inclusion process between the teachers of both locations, as well as variations in the perception of the dimensions proposed. It was concluded that the existence of differences in the perception of inclusion of the teachers of the two districts has been due to the positions of the teachers regarding the institutional policy and their perception of the inclusion process.

Keywords: inclusion, inclusive policies, inclusive culture, diversity, equal opportunities

\section{Introducción}

Los derechos fundamentales de todas las personas incluyen el acceso a una serie de servicios básicos que garantizan la realización personal. Uno de estos derechos es el acceso a una educación de calidad, lo que garantiza que todas las personas tengan las mismas oportunidades de aprendizaje. Por lo tanto, la atención a la diversidad en el aula debe ser uno de los principios de la educación superior, que conduzca a la implementación de acciones destinadas a garantizar que todas las personas, independientemente de sus características, tengan acceso a la igualdad de oportunidades en el proceso educativo (Done \& Murphy, 2016). Es una obligación legal de las instituciones educativas garantizar la igualdad de oportunidades para todos los estudiantes, y 
esperamos que este estudio contribuya a mejorar el éxito académico de los estudiantes con discapacidades y a promover su inclusión académica real. (Kiuppis, 2014; Calderón, 2014)

El trasunto de la cuestión es abordado desde un cambio de paradigma, tal como lo sugiere Saloviita (2017); por ello el nuevo paradigma inclusivo causó revuelo sobre el desafío del cambio en el entorno social, al insistir en que, en primer lugar, es deber de las escuelas dar cabida a las diversas necesidades de los niños. Las escuelas deben adaptarse para poder acoger a todos los niños en las aulas ordinarias, incluidos los más discapacitados (Armstrong Armstrong \& Barton, 2016; Arnaiz \& Azorín, 2014; Norwich, 2014, Arnaíz, 2012; McCowan, 2011; Magnus, 2010). Así pues, el enfoque del nuevo concepto se centró en las discapacidades, y especialmente en las discapacidades graves.

Evaluar el proceso de inclusión desde la perspectiva occidental de corte neoliberal no es una tarea sencilla de asumir debido a la amplitud de aristas complejas que tal asunto atañe. Al respecto Done \& Murphy (2016) mencionaron que el concepto de inclusión como participación, pone de relieve las tensiones entre dos vertientes (performance competencia) y los discursos relativos al profesionalismo y por ello demandan que la agenda de posibles cambios deberá contemplar estrategias discursivas que eviten o compliquen asumir el proceso inclusivo como un derecho y no una asistencia en el campo de la sociedad de libre mercado. Por su parte Duke y Morillo mencionaron respecto al rasgo dilemático en relación a la inclusión se sustenta en la complejidad que posee y la amplia problemática que engloba en relación a las opciones que se tenga a bien disponer en cualquier instancia "derivando en efectos más o menos deseables o favorables, dependiendo de las variables contextuales y culturales que entran en juego para su resolución.” (2016, p.13).

El asunto es más relevante en la actualidad. Haug (2016) dio a conocer que en todos los países parece existir una brecha entre las formulaciones y las realizaciones de la educación inclusiva. Sin embargo, el reto en la educación inclusiva en la praxis son las dificultades de aplicación (Lakkala \& Thuneberg, 2018). De lo anterior se tiene que es un hecho las múltiples diferencias entre las naciones respecto a la praxis de la inclusión en el campo educativo; pero es un hecho también que en ninguna de ellas ha logrado construir una escuela que está a la altura de los ideales e intenciones de inclusión (Rana \& Culbreath, 2018, Armstrong, Armstrong \& Barton, 2016; Verdugo \& Rodríguez, 2013).

Cuando las capacidades de un estudiante no se correspondían con las demandas del entorno, el viejo paradigma buscaba una solución por parte del estudiante; se esperaba que el estudiante cambiara para poder calificar para el entorno regular (Rayner, 2017; Parra, 2011; Lumby \& Morrison, 2009). Al respecto Rayner (2017) hizo un análisis circunspecto sobre las admisiones escolares, refiriéndose a las mismas con un eje clave donde las preocupaciones sobre la equidad y la inclusión educativa interactúan frente a las perspectivas sobre el posicionamiento en el mercado y los resultados educativos. Por ello se estimó que las recientes reformas escolares se han construido discursivamente como un medio, no sólo de mejorar la calidad de la educación, sino también de realizar una labor de justicia social (Andrade, 2011).

Por otro lado, al tratar los diversos flancos que merece la atención de la inclusión, Subramaniam \& Subramaniam (2017), tuvieron a bien en deslindar acerca de las divisiones creadas por las desigualdades de ingresos, el crecimiento desequilibrado y el círculo vicioso de la pobreza han atrapado el aprendizaje y retrasado las estrategias previstas para la inclusión educativa. Por su parte, Rana \& Culbreath (2018), plantearon que el actual discurso de la 
globalización ha dado forma al mundo de la educación de la primera infancia a medida que la sociedad se ha ido diversificando cada vez más. A medida que la población se hace más diversa, los maestros se ven obligados a incorporar pedagogías inclusivas.

\section{Aspectos teóricos}

\section{Acerca de la inclusión en educación}

En el ámbito de la educación, la última década del siglo XX y la primera del siglo XXI se han caracterizado por el dinamismo y la nueva configuración del aula. La globalización y la velocidad de los cambios sociales, económicos, culturales y tecnológicos plantean nuevas exigencias a las escuelas que obligan a los sistemas educativos a renovarse constantemente para responder a las demandas y necesidades de las personas y las sociedades (Rodríguez \& Caro, 2016). La pedagogía culturalmente inclusiva se centra en el respeto de las diferencias culturales y la inclusión de la diversidad (Arroyo, 2018; Rana \& Culbreath, 2018; Hernández \& Tobón, 2016; Granada, Pomés \& Sanhueza, 2013, Parra, 2011). También hace hincapié en la equidad y la justicia en el empoderamiento del individuo como rol social (Rodríguez y Caro, 2016; Cifuentes, 2016). La pedagogía culturalmente inclusiva no es un concepto nuevo (Martins, Borges \& Gonçalves, 2016). Se ha considerado ampliamente como una estrategia útil para mejorar los resultados educativos de aquellos estudiantes con menos oportunidades en todo el mundo (Rodríguez y Caro, 2016). Un punto de vista particular es lo expuesto por Messiou (2016), acerca del hecho de que la educación inclusiva puede entenderse de diferentes maneras, según los diferentes autores y partes interesadas puedan dar diferentes posturas al respecto.

De acuerdo con lo manifestado por Bešić, Paleczek, Krammer \& Gasteiger (2017), habrá que deslindar entre la educación integral y la inclusión en la educación; por ello explicaron que la principal diferencia es que en la educación integrada se hace hincapié en que el estudiante se adapte al sistema escolar existente y no en que el sistema se adapte a las necesidades individuales de los estudiantes; mientras que en la educación inclusiva se hace hincapié en la necesidad de cambiar el sistema escolar para satisfacer las necesidades de todos los estudiantes. Por su parte Haug (2016) precisó que la inclusión implica el derecho a la educación para todos los estudiantes; además estimó que los valores asociados a la inclusión tienen vínculos con la ideología interaccionista y giran en torno al compañerismo, la participación, la democratización, el beneficio, la igualdad de acceso, la calidad, la equidad y la justicia. Por su parte, Saloviita (2017) mencionó que la inclusión en sí misma no se trataba de una nueva palabra que se estaba introduciendo, sino de una nueva forma de pensar sobre la relación entre las capacidades individuales y las características del medio ambiente.

El proceso de inclusión en relación al campo de la educación, es un asunto en esencia complejo de compilar, ya que aborda una amplia terminología con una gama diversa de significados que se adecuan en función del contexto y la coyuntura que se ha generado en torno a su desarrollo y aplicación (Roberts y Cifuentes, 2016). Según lo manifestado por Duke y Morillo (2016) la idea en torno a la inclusión educativa no se encuentra bien delimitada, siendo algunas veces algo ambiguo y en otras ocasiones podría generar una serie de controversias y dilemas; debido al cómo ha evolucionado y extendiéndose progresivamente, tanto así hasta convertirse en un anhelo al cual muchos países han virado su agenda y los discursos políticos, desde la década de los noventa, para atender esta problemática, de forma especial en lo que respecta al sistema educativo y sus diversos estamentos (Ndemanu \& Jordan, 2018). 
Respecto a la situación de la inclusión en el nivel superior, se tiene una situación particular en la que se reflejan procesos de cambio (Canchala, Serrato \& Criollo, 2014; Verdugo \& Rodríguez, 2013), ya que en la actualidad las mismas no están reservadas para una élite, por lo que deben garantizar la igualdad de oportunidades para los estudiantes con discapacidades adoptando la heterogeneidad social, cultural y lingüística que caracteriza a la sociedad (Rayner, 2017; Martins, Borges \& Gonçalves, 2017; Florian, Black \& Rouse, 2017, García y Espinoza, 2013). Idealmente, las instituciones de educación superior deberían estar estructuradas de tal manera que promuevan la participación activa de los estudiantes con discapacidades en el proceso de enseñanza y aprendizaje y para crear las condiciones adecuadas para que terminen sus cursos (Melo y Martins, 2016; Pérez \& Memije, 2015; Talero Mahecha \& Cubillos, 2014).

Existen estudios que han investigado la forma en que aspectos negativos específicos como el racismo, los prejuicios y la discriminación afectan al desarrollo de la autonomía de los estudiantes en los contextos de la escuela y el aula (Kumar, Zusho \& Bondie, 2018); hecho que también se guarda relación entre la competencia cultural y académica tanto de los profesores como de los estudiantes. Las tendencias ligadas a la exclusión social por patrones étnicos es una cruda realidad en diversas locaciones en el globo y que, a pesar de las conquistas en los derechos universales del ser humano, aquello que ha convertido en una serie tara que genera una secuela muy negativa en la sociedad y uno de los estamentos sociales donde puede darse cuadros de esa categoría son las escuelas, las que solo reflejan lo contextualizado en su entorno (Tosi, Peláez, Larrañaga, Saavedra, \& Baeza, 2016; Martins, Borges \& Gonçalves, 2016). Por ello Arroyo (2018, p.145) tuvo a bien a establecer que "En muchas ocasiones, las diferencias en nuestras escuelas sirven para justificar la exclusión educativa y, por ende, social de muchos niños y niñas."

La importancia de comprometerse con la identidad cultural y la alteridad de los profesores y cómo se relacionan con los niños, su propia cultura y la de sus compañeros como procesos que orientan a consolidar la inclusividad en los niños (Rana \& Culbreath. 2018). Nosotros argumentamos que además de comprometerse con la alteridad cultural de los maestros, también hay la necesidad de mirar más allá de los enfoques universalizadores de la alteridad y de pasar hacia una pedagogía culturalmente inclusiva y relevante.

Con relación a las políticas de inclusión educativa, Torres, J. A., \& Fernández (2015), han manifestado que, en la última década, los aspectos relacionados a la educación inclusiva han logrado conseguir un crecimiento y reconocimiento continuo en diversos estamentos internacionales, nacionales y de forma especial en las reformas escolares.

\section{Metodología}

Debido a la coyuntura particular de la presente investigación, se ha desarrollado la metodología descriptiva-comparativa, la cual se ejecutó en procedimientos definidos y secuenciados como determinación, recopilación, análisis, identificación, elaboración y desarrollo (Hernández y Mendoza, 2018); todo ello con la finalidad de poder hacer la descripción y comparación realidades diferentes frente a un parámetro definido de trabajo. De acuerdo con lo anteriormente mencionado, se optó por realizar la compilación conveniente de la mayor cantidad de data relevante, valiéndose para ello de la metodología hipotético-deductiva. A fin de poder solventar lo planificado se optó por la colecta de datos empleando como instrumento el 'Index For Inclusión' desarrollado por Ainscow y Booth (2002), el cual constó de las siguientes dimensiones: 'Creación de culturas inclusivas' (con dos indicadores y 13 ítems), 'Creación de políticas 
inclusivas' (con tres indicadores y 3 indicares y 15 ítems), y 'Desarrollo de prácticas inclusivas' (con dos indicadores y 18 ítems), donde las respuestas se acumulan bajo escala de tipo Likert. A su vez los detalles de la población se detallan en la tabla 1.

Tabla 1. Población estudiada.

\begin{tabular}{ccc}
\hline Distrito & Institución Educativa & Población \\
\hline Los Olivos & 3084 Enrique Guzmán y Valle & 60 \\
San Martín de Porres & 2088 República Federal de Alemania & 60 \\
\hline
\end{tabular}

\section{Resultados}

La percepción del proceso relacionado con la inclusión se muestra en la Tabla 2, donde se hacen las comparaciones para ambos distritos estudiados: Los Olivo y San Martín de Porres; de lo cual se rescata que en el nivel 'Bueno' se obtuvo la mayor puntuación para ambos distritos $(63,3 \%$ y $65 \%$ respectivamente), que según la opinión de los encuestados nos arroja un 64,2\% del total de las encuestas realizadas, lo que ya de por sí nos da una estimación considerable. Otro aspecto que se rescata de dicha tabla es el hecho de que la percepción de un nivel regular por parte de los docentes de ambos distritos es bastante baja $(1,7 \%)$ y que la situación avizora como positiva ya que en promedio de un $34 \%$ por ambas partes ha estimado que es 'muy bueno' el proceso de inclusión que se ha desarrollado en ambos Distritos.

Tabla 2.

Comparación porcentual de los niveles del proceso de inclusión en educación básica regular en los docentes de los distritos de Los Olivos y San Martin de Porres.

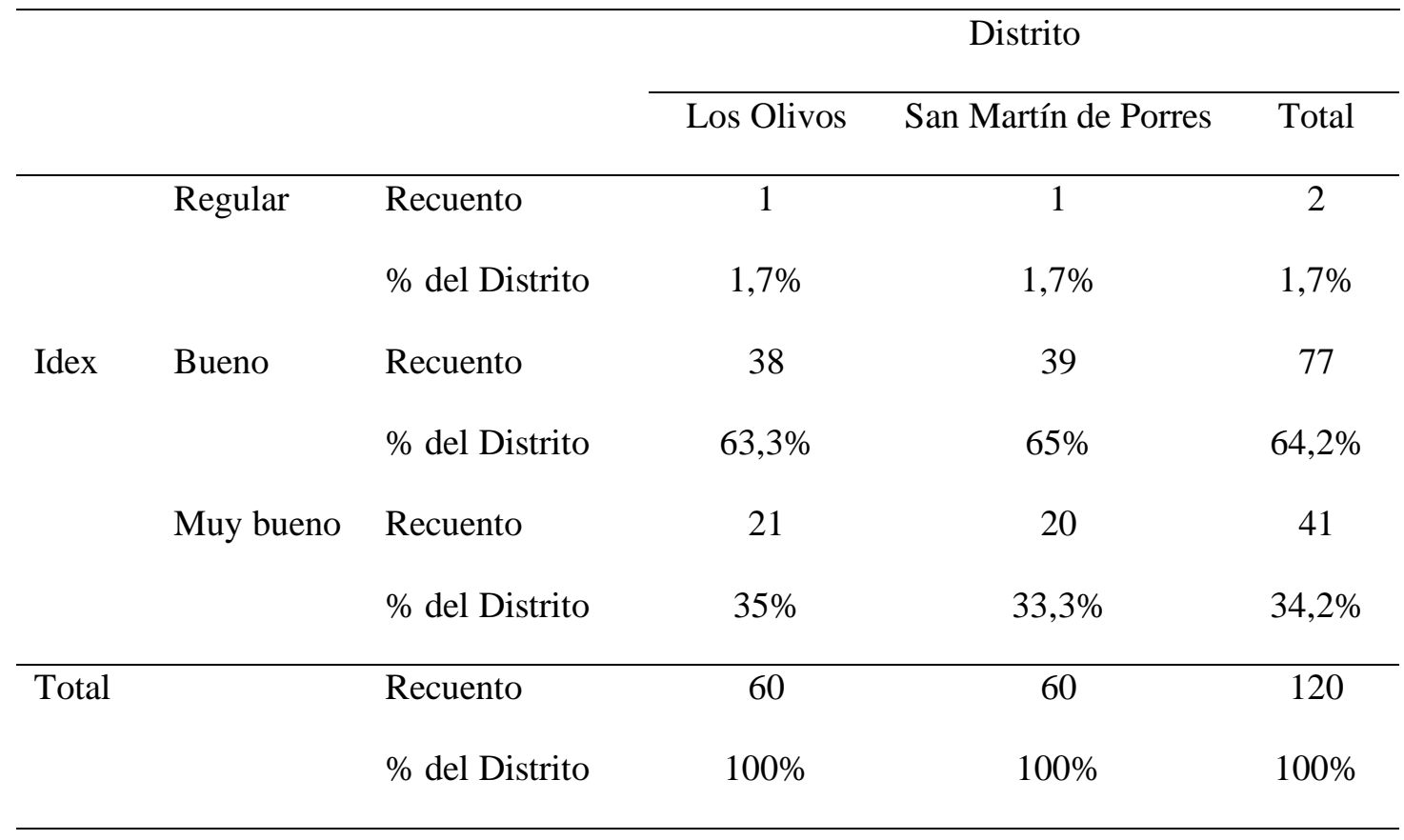


Respecto a la evaluación de la percepción para cada una de las dimensiones señaladas se muestran en la Tabla 3. Al respecto para la primera dimensión denominada 'Creación de culturas inclusivas' se tiene que las estimaciones de mayor porcentaje se ubican en el nivel ¿Bueno', siendo de 63,3\% y 66,7\% para los distritos de Los Olivos y San Martín de Porres respectivamente. De dicha dimensión también se rescata el porcentaje cuasi similar para la misma dimensión respecto a la percepción del nivel ‘Muy Bueno' por parte de los docentes de ambos distritos.

Seguidamente para la dimensión denominada 'Creación de las políticas inclusivas', los datos obtenidos que se muestran en la Tabla reflejan que en el Distrito de los Olivos se halló una mejor aceptación de la dimensión evaluada en el nivel 'Bueno' respecto al de Los Olivos, con una diferencia del 15\% entre ambos Distritos. Sin embargo, para el nivel 'Muy Bueno' son los docentes del Distrito de San Martín de Porres los que muestran una mayor aceptación de la percepción mencionada con un porcentaje 46,7\% respecto al de Los Olivos, cuyos docentes reportaron solo un $30 \%$.

Finalmente, para la dimensión 'Desarrollo de prácticas inclusivas' los datos mostrados en la Tabla mostraron que los docentes del Distrito de San Martín de Porres mostraron una mejor percepción de dicha dimensión en lo que se refiere al nivel 'Bueno' ya que el 61,7\% de los mismos se identificó con dicho nivel respecto al $51,7 \%$ de los docentes del Distrito de Los Olivos. Seguidamente para el nivel 'Muy Bueno' para la dimensión mencionada, la situación se revierte ya que son los docentes de Los Olivos quienes en un 41,7\% muestra su identificación para la dimensión mencionada respecto al 36,7\% de los docentes de Los Olivos, estimándose tal diferencia en un $10 \%$ aproximadamente.

Tabla 3.

Niveles de frecuencia comparativa de los niveles de la variable 'Proceso de inclusión' y sus respectivas dimensiones en los docentes de los distritos de Los Olivos y San Martin de Porres.

\begin{tabular}{llllccc}
\hline & & & & \multicolumn{3}{c}{ Distrito } \\
\cline { 4 - 6 } & & & Los Olivos & San Martín de Porres & Total \\
\hline Creación & de & culturas & Regular & $1,7 \%$ & $1,7 \%$ & $1,7 \%$ \\
inclusivas & & & Bueno & $63,3 \%$ & $66,7 \%$ & $65 \%$ \\
& & & Muy Bueno & $35 \%$ & $31,7 \%$ & $33,3 \%$ \\
Creación & de & \multirow{2}{*}{ políticas } & Regular & $3,3 \%$ & $1,7 \%$ & $2,5 \%$ \\
inclusivas & & & Bueno & $66,7 \%$ & $51,7 \%$ & $59,2 \%$ \\
& & & Muy Bueno & $30 \%$ & $46,7 \%$ & $38,3 \%$ \\
Desarrollo & de & prácticas & Regular & $6,7 \%$ & $1,7 \%$ & $4,2 \%$ \\
inclusivas & & & Bueno & $51,7 \%$ & $61,7 \%$ & $56,7 \%$ \\
& & & Muy Bueno & $41,7 \%$ & $36,7 \%$ & $39,2 \%$ \\
\hline Total & & & & $100 \%$ & $100 \%$ & $100 \%$
\end{tabular}


A fin de desarrollar la comparación estadística de las percepciones relativas al proceso de inclusión para ambas instituciones estudiadas, se aplicó el estadístico no paramétrico de la U Mann de Whitney, asumiendo que el nivel significativo de prueba de $\alpha=, 05$ frente al valor de significación estadística de p valor. Dicha prueba va a confirmar si existieron diferencias en el nivel de percepción del proceso de inclusión en educación básica regular en los docentes del nivel primario de Lima Norte; así como también establecer si dichas diferencias ocurrieron también para cada una de las dimensiones mencionadas en el presente estudio.

De acuerdo con la información presentada en la Tabla 4 para la evaluación de los valores inferenciales para la comparación de la variable 'Proceso de inclusión' entre los docentes de los Distritos de Los Olivos y San Martín de Porres se obtuvo que no ocurrieron diferencias significativas entre ambos grupos al respecto ya que el valor de $\mathrm{Zc}$ fue menor a ,05 $(-, 186)$. De forma similar se dieron resultados similares para las dimensiones 'Creación de culturas inclusivas' (-,373), 'Creación de políticas inclusivas' (-1,902), 'Desarrollo de prácticas inclusivas' (-,190), todos ellos menores a ,05 por lo que se establece que no existieron diferencias significativas en las percepciones de los docentes de ambos Distritos evaluados en las dimensiones indicadas.

Tabla 4.

Significancia del nivel de percepción del proceso de inclusión en educación en los docentes de Los Olivos y San Martín de Porres. (variable y dimensiones).

\begin{tabular}{|c|c|c|c|c|c|c|}
\hline & & & & Rangos & & \\
\hline & & & $\begin{array}{c}\text { Rango } \\
\text { promedio }\end{array}$ & $\begin{array}{c}\text { Suma } \\
\text { de } \\
\text { rangos }\end{array}$ & Paráme & \\
\hline Proceso de & Los Olivos & 60 & 60,99 & 3659,50 & $\mathrm{Zc}$ &,- 186 \\
\hline inclusión & San Martín de Porres & 60 & 60,01 & 3600,50 & Sig. asim. &, 853 \\
\hline & Total & 120 & & & & \\
\hline Creación de & Los Olivos & 60 & 61,48 & 3689,00 & $\mathrm{Zc}$ &,- 373 \\
\hline culturas & San Martín de Porres & 60 & 59,52 & 3571,00 & Sig. asim. & ,709 \\
\hline & Total & 120 & & & & \\
\hline Creación de & Los Olivos & 60 & 55,32 & 3319,00 & $\mathrm{Zc}$ & $-1,902$ \\
\hline políticas & San Martín de Porres & 60 & 65,68 & 3941,00 & Sig. asim. &, 057 \\
\hline & Total & 120 & & & & \\
\hline $\begin{array}{l}\text { Desarrollo de } \\
\text { prácticas }\end{array}$ & Los Olivos & 60 & 61,03 & 3661,50 & $\mathrm{Zc}$ &,- 190 \\
\hline inclusivas & San Martín de Porres & 60 & 59,98 & 3598,50 & Sig. asim. & ,849 \\
\hline & Total & 120 & & & & \\
\hline
\end{tabular}




\section{Discusión}

Según los resultados obtenidos para la evaluación de percepción del proceso de inclusión en educación básica regular entre los docentes de los distritos de Los Olivos y San Martín de Porres, se tiene que no ocurrieron diferencias significativas. Según la comparación porcentual, se tiene que los docentes de ambas locaciones percibieron que el proceso de inclusión no es el más idóneo $(1,7 \%)$, debido a desventajas manifiestas en las aulas, ya sea por aspectos conductuales o por los mismos procesos cognitivos de los estudiantes. Aunque dicho resultado es muy bajo, se debería tener en cuenta para investigaciones futuras el poder ahondar en tal asunto a fin de poder dilucidar aspectos que, por la naturaleza del presente estudio, escapan a sus objetivos.

En los resultados obtenidos para la dimensión 'Culturas inclusivas', se halló una diferencia del 3,4\% en la comparación para el nivel 'Bueno' en relación a los docentes de ambos distritos estudiados; siendo dicha diferencia cuasi similar (3,4\%) en relación al nivel 'Muy bueno' lo que se traduce en una homogeneidad en relación con dos jerarquías cualitativas diferentes, que entre otras cosas tendría que asociarse al patrón socioeconómico de los docentes de ambas locaciones, ya que no hay muchas diferencias en dicho contexto debido a su proximidad geográfica y además la ubicación en una zona populosa de la ciudad. El resultado logrado se aproxima a lo referenciado por Arroyo (2018) quien concluyó que el proceso de inclusión en la dimensión de cultura inclusiva se vería garantizada si en las aulas se tomara en cuenta la importancia de la diversidad y multiculturalidad de los componentes de una zona dada.

Del análisis de la dimensión 'Políticas Inclusivas', se obtuvo como pertinente los valores porcentuales para el nivel 'Bueno' siendo de 66,7\% para los docentes del Distrito de Los Olivos frente a un 51,7\% para los de San Martín de Porres, estableciéndose una diferencia del 15\%, lo que refleja una mayor aceptación de tales políticas en los docentes de Los Olivos. Respecto al nivel 'Muy bueno', se presentó una diferencia porcentual del 16,7\%, para los docentes de las locaciones indicadas, teniendo en este caso una inversión de la percepción respecto al rango anterior, ya que son los docentes del Distrito de San Martín de Porres quienes en un mayor porcentaje han percibido que hubo un mejor desarrollo de las políticas inclusivas en sus instituciones. Lo anterior se pudo relacionar con el aporte encontrado por Hernández y Tobón (2016), quienes manifestaron la urgencia de la generación de una propuesta curricular orientada a la atención de las necesidades de un proceso inclusivo continuo a fin de prodigar una educación con equidad, bajo parámetros éticos y con responsabilidad. Por su parte Rayner (2017), destacó que el caso de la política de admisión de una escuela revela cómo los profesores, el resto del personal y los que desempeñan funciones de liderazgo responden a los cambios a nivel organizativo y personal; lo que se traduce en la displicencia de dichos profesionales estará limitada por las exigencias del régimen regulador y el posicionamiento en el mercado.

Finalmente, en lo que respecta a la dimensión 'Prácticas Inclusivas', los resultados que se generaron para el rango 'Bueno' mostraron una diferencia porcentual del 10\%, que de acuerdo al análisis respectivo se concluyó que los docentes del Distrito de San Martín de Porres han desarrollado de forma más óptima los aspectos relacionados a las prácticas de inclusividad en sus instituciones. Dicha tendencia, aunque con un porcentaje algo menor (5\%), se revirtió en el rango 'Muy bueno' ya que los docentes de Los Olivos percibieron que dichas prácticas fueron mucho más pertinentes en sus respectivas instituciones. Al respecto la investigación reportada por Lakkala \& Thuneberg (2018) proporcionó información sobre las diferencias entre los profesores con respecto al nivel de formación en el servicio para la enseñanza de aulas inclusivas con respecto a sus actitudes acerca de la inclusión, ya que sus resultados muestran que la capacitación 
inclusiva en el servicio tiene un efecto positivo impacto en las actitudes de los profesores. Por ello Saloviita (2017) recomienda que debe tenerse muy en cuenta la formación en el servicio, así como la orientación y la planificación de las disposiciones de enseñanza inclusiva proporcionan a los profesores un sentido de competencia para tener éxito enseñan a diversos alumnos, reforzando así su autoeficacia en la educación inclusiva.

De forma coyuntural, compartimos la perspectiva de Martins et al. (2017) quienes refrendaron el hecho que, si las instituciones tienen como objetivo garantizar la igualdad de oportunidades para todos los estudiantes, las mismas deberán de abolir toda forma de discriminación y redefinir estrategias para garantizar la participación efectiva de los estudiantes con discapacidad, incluyendo aspectos clave como accesos, inscripción y la culminación exitosa de un programa académico. Así mismo el estudio se orienta a las propuestas de Done \& Murphy (2016) quienes sugirieron la variación del discurso orientado a los cambios, sustentado en la literatura sobre inclusión educativa, que busca que se aliente a los maestros, que se muestran escépticos ante la posibilidad de que las iniciativas de políticas de inclusión generen justicia social o, que cuestionan la motivación política que subyace a dichas iniciativas, a que se identifiquen con la imagen potencialmente seductora del maestro como agente de cambio (Durán \& Giné, 2017), es decir, como un profesional que puede, con el tiempo, provocar el cambio social y crear el entorno educativo deseado, aunque ello signifique el cumplimiento a corto plazo de directivas políticas problemáticas.

Por otro lado, la apuesta inclusiva deberá ir de la mano no solo con la permisibilidad de los grupos postergados en el régimen social; sino que además deberá tenerse en cuenta un punto básico que tenuemente se ha enfocado, tal como es el acceso a la tecnología, motivo por el cual concordamos con lo manifestado por Subramaniam \& Subramaniam (2017) quienes manifestaron que el desarrollo de habilidades en los grupos postergados, permitirá aprovechar las oportunidades y aprovechar la tecnología para simplificar el alcance inclusivo ideal, valiéndose de métodos innovadores que permitirán liberar el potencial sin explotar y, por lo tanto, elevar los estilos de vida al empoderar hábilmente.

\section{Referencias}

Ainscow, M. (2002). Understanding the development of inclusive schools. Routledge. New York. https://doi.org/10.4324/9780203016619

Andrade-Ruiz, F. (2011). La inclusión educativa en el aula regular: un caso de Síndrome de Asperger. Revista Electrónica Educare, (15), 39-53. https://bit.ly/2Br3Oqg

Armstrong, F., Armstrong, D., \& Barton, L. (2016). Inclusive education: Policy, contexts and comparative perspectives. Routledge. England.

Arnaiz-Sánchez, P. A. (2012). Escuelas eficaces e inclusivas: cómo favorecer su desarrollo. Educatio Siglo XXI, 30(1), 25-44. https://bit.ly/3egC2vv

Arnaiz-Sánchez, P. A., \& Azorín-Abellán, C. M. (2014). Autoevaluación docente para la mejora de los procesos educativos en escuelas que caminan hacia la inclusión. Revista colombiana de Educación, (67), 227-245. https://bit.ly/2YMfQmi 
Arroyo-González. M. J. (2018). La Educación Intercultural: un camino hacia la inclusión educativa. Revista de Educación Inclusiva, 6(2), 144-159.

Bešić, E., Paleczek, L., Krammer, M., \& Gasteiger-Klicpera, B. (2017). Inclusive practices at the teacher and class level: the experts' view. European Journal of Special Needs Education, 32(3), 329-345. https://doi.org/10.1080/08856257.2016.1240339

Calderón, M. (2014). La educación inclusiva es nuestra tarea. Educación. 21(40) 43-58. https://bit.ly/30ZCHxh

Canchala-Obando, M., Serrato-Mendoza, M. P., \& Criollo-Castro, C. (2014). Diagnóstico sobre inclusión educativa en discapacidad en los programas de pregrado de la Universidad Mariana. Boletín Informativo CEI, 1(2), 92-95. https://bit.ly/2YRFiGV

Cifuentes-Garzón, J. E. (2016). Inclusão e identidade das práticas discursivas dos alunos na escola. Revista Educación y Desarrollo Social, 10(9), 78-97. http://dx.doi.org/10.18359/reds.1450

Done, E. J., \& Murphy, M. (2016). The responsibilisation of teachers: a neoliberal solution to the problem of inclusion. Discourse: Studies in the Cultural Politics of Education, 39(1), 142155. doi:10.1080/01596306.2016.1243517

Duk, C., \& Murillo, F. J. (2016). La inclusión como dilema. Revista Latinoamericana de Educación Inclusiva, 10(1), 11-14. https://bit.ly/2YD3w8Z

Durán-Gisbert, D., \& Giné-Giné, C. (2017). La formación del profesorado para la educación inclusiva: Un proceso de desarrollo profesional y de mejora de los centros para atender la diversidad. Revista Latinoamericana de Educación Inclusiva, 1(1), 153-170. https://bit.ly/30UDXlz

Florian, L. Black-Hawkins, K., \& Rouse, M. (2017). Achievement and Inclusion in Schools. Second edition. Routledge. New York.

García-García, C. \& Espinoza, Ó. (2013). Equidad e inclusividad en la educación superior en los países andinos: los casos de Bolivia, Chile, Colombia y Perú. Revista de educación Superior, 42(2), 137-144. https://bit.ly/2NdoAfP

Granada-Azcárraga, M., Pomés-Correa, M. P., \& Sanhueza-Henríquez, S. (2013). Actitud de los profesores hacia la inclusión educativa. Papeles de Trabajo, (25), 51-59. https://bit.ly/2YfvxDj

Haug, P. (2017). Understanding inclusive education: ideals and reality. Scandinavian Journal of Disability Research, 19(3), 206-217. https://doi.org/10.1080/15017419.2016.1224778

Hernández-Sampieri, R., y Mendoza-Torres, C. P. (2018). Metodología de la investigación. 1a. ed. Mc Graw-Hill-Interamericana. México.

Hernández-Ayala, H., \& Tobón-Tobón, S., (2016). Análisis documental del proceso de inclusión en la educación. Ra Ximhai, 12(6), 399-420. https://bit.ly/2AQK7sd 
Kiuppis, F. (2014). Why (Not) Associate the Principle of Inclusion with Disability? Tracing Connections from the Start of the 'Salamanca Process'. International Journal of Inclusive Education 18(7): 746-761. doi:10.1080/13603116.2013.826289

Kumar, R., Zusho, A., \& Bondie, R. (2018). Weaving Cultural Relevance and Achievement Motivation Into Inclusive Classroom Cultures. Educational Psychologist, 53(2), 78-96. doi:10.1080/00461520.2018.1432361

Lakkala, S., \& Thuneberg, H. (2018). Teachers' perceptions of educational reform aimed at inclusion. Education in the North, 25(1-2), 295-319. https://bit.ly/3dDakYz

Lumby, J., \& Morrison, M. (2009). Youth perspectives: schooling, capabilities frameworks and human rights. International Journal of Inclusive Education, 13(6), 581-596. https://doi.org/10.1080/13603110801995920

Magnus-Reindal, S. (2010). What is the purpose? Reflections on inclusion and special education from a capability perspective. European Journal of Special Needs Education, 25(1), 1-12. https://doi.org/10.1080/08856250903450806

Martins, M. H., Borges, M. L., \& Gonçalves, T. (2017). Attitudes towards inclusion in higher education in a Portuguese university. International Journal of Inclusive Education, 22(5), 527-542. doi:10.1080/13603116.2017.1377299

Martins, M. H., Borges, M. L. \& Gonçalves, T. (2016). Legislation for Higher Education Students with Disabilities in Brazil and Portugal: Some Reflections. Acta Scientiarum, Education 38(3): 259-269. https://doi.org/10.1080/13603116.2017.1377299

McCowan, T. (2011). Human rights, capabilities and the normative basis of 'Education for All'. Theory and Research in Education, 9(3), 283-298. https://doi.org/10.1177/1477878511419566

Messiou, K. (2016). Research in the field of inclusive education: time for a rethink? International Journal of Inclusive Education, 21(2), 146-159. doi:10.1080/13603116.2016.1223184

Ndemanu, M. T., \& Jordan, S. (2018). Culturally responsive pedagogy for African immigrant children in U.S. P-12 schools. Journal of Black Studies, 49(1), 71-84. https://doi.org/10.1177/0021934717736065

Norwich, B. (2014). How does the capability approach address current issues in special educational needs, disability and inclusive education field? Journal of Research in Special Educational Needs, 14(1), 16-21. https://doi.org/10.1111/1471-3802.12012

Parra-Dussan, C. (2011). Educación inclusiva: un modelo de diversidad humana. Educación y desarrollo social, 5(1), 139-150.

Pérez-López, A. L., \& Memije-Alarcón, N. Y. (2015). Educación con inclusión en la Universidad Autónoma de Guerrero. Reencuentro, (70), 161-170. https://bit.ly/31axljd

Rana, L., \& Culbreath, Y. (2018). Culturally inclusive pedagogies of care: A narrative inquiry. Journal of Pedagogy, 10(2), 87-99. doi: 10.2478/jped-2019-0008 
Rayner, S. M. (2017). Admissions policies and risks to equity and educational inclusion in the context of school reform in England. Management in Education, 31(1), 27-32. https://doi.org/10.1177/0892020616687699

Roberts-Jorquera, K., \& Cifuentes Garzón, J. E. (2016). Inclusión e identidad desde las prácticas discursivas de los estudiantes en la escuela. Revista Educación y Desarrollo Social, 10(1), 78-97. https://doi.org/10.18359/reds. 1450

Rodríguez, R. C., \& Caro, T. T. (2016). Cultural Diversity and Socio-Educational Inclusion. Pedagogía Social. Revista Interuniversitaria, (29), 21-24. https://bit.ly/2ZgOvZx

Saloviita, T. (2017). How common are inclusive educational practices among Finnish teachers? International Journal of Inclusive Education, 22(5), 560-575. doi:10.1080/13603116.2017.1390001

Subramaniam, S., \& Subramaniam, R. (2017). Leveraging Technology for Educational Inclusion. Universal Journal of Educational Research 5(1), 94-98. doi: 10.13189/ujer.2017.050111

Talero-Parra, A. D., Mahecha, L. D., \& Cubillos, N. (2014). Acceso de personas con discapacidad a la formación superior desde la perspectiva de terapia ocupacional. Revista Colombiana de Rehabilitación, 13(1), 88-95.

Torres, J. A., \& Fernández, J. M. (2015). Promoviendo escuelas inclusivas: análisis de las percepciones y necesidades del profesorado desde una perspectiva organizativa, curricular y de desarrollo profesional. Revista Electrónica Interuniversitaria De Formación Del Profesorado, 18(1), 177-200. https://doi.org/10.6018/reifop.18.1.214391

Tosi, I., Peláez, L., Larrañaga, M., Saavedra, E., \& Baeza, E. (2016). Educación inclusiva y vida independiente. Universitas. Revista de Filosofía, Derecho y Política, (24), 134-164. http://dx.doi.org/10.20318/universitas.2016.3178

Verdugo-Alonso, M. Á., \& Rodríguez-Aguilella, A. (2013). Valoración de la inclusión educativa desde diferentes perspectivas. Revista Española sobre Discapacidad Intelectual, 39(3), 525. https://bit.ly/2zJJr7k 